\section{Genomic history of the seventh pandemic of cholera in Africa}

François-Xavier Weill, ${ }^{1,2 *}$ Daryl Domman, ${ }^{2}$ Elisabeth Njamkepo, ${ }^{1}$ Cheryl Tarr, ${ }^{3}$ Jean Rauzier, ${ }^{1}$ Nizar Fawal, ${ }^{1}$ Karen H. Keddy, ${ }^{4,5}$ Henrik Salje, ${ }^{6,7}$ Sandra Moore, ${ }^{8}$ Asish K. Mukhopadhyay, ${ }^{9}$ Raymond Bercion, ${ }^{10,11}$ Francisco J. Luquero, ${ }^{12}$ Antoinette Ngandjio, ${ }^{13}$ Mireille Dosso, ${ }^{14}$ Elena Monakhova, ${ }^{15}$ Benoit Garin,,${ }^{11}+$ Christiane Bouchier, ${ }^{16}$ Carlo Pazzani, ${ }^{17}$ Ankur Mutreja, ${ }^{18,19}$ Roland Grunow, ${ }^{20}$ Fati Sidikou, ${ }^{21}$ Laurence Bonte,${ }^{22} \ddagger$ Sébastien Breurec,${ }^{10}$ Maria Damian,,${ }^{23}$ Berthe-Marie Njanpop-Lafourcade, ${ }^{24}$ Guillaume Sapriel, ${ }^{25,26}$ Anne-Laure Page, ${ }^{12}$ Monzer Hamze, ${ }^{27}$ Myriam Henkens, ${ }^{28} \ddagger$ Goutam Chowdhury, ${ }^{9}$ Martin Mengel, ${ }^{24}$ Jean-Louis Koeck, ${ }^{29} \S$ Jean-Michel Fournier, ${ }^{30}$ Gordon Dougan, ${ }^{2,18}$

Patrick A. D. Grimont, ${ }^{31}$ Julian Parkhill, ${ }^{2}$ Kathryn E. Holt, ${ }^{32}$ Renaud Piarroux, ${ }^{8}$ Thandavarayan Ramamurthy, ${ }^{19}$ Marie-Laure Quilici, ${ }^{1,30}||$ Nicholas R. Thomson ${ }^{2,33}||$

The seventh cholera pandemic has heavily affected Africa, although the origin and continental spread of the disease remain undefined. We used genomic data from 1070 Vibrio cholerae $\mathrm{O} 1$ isolates, across 45 African countries and over a 49-year period, to show that past epidemics were attributable to a single expanded lineage. This lineage was introduced at least 11 times since 1970, into two main regions, West Africa and East/Southern Africa, causing epidemics that lasted up to 28 years. The last five introductions into Africa, all from Asia, involved multidrug-resistant sublineages that replaced antibiotic-susceptible sublineages after 2000 . This phylogenetic framework describes the periodicity of lineage introduction and the stable routes of cholera spread, which should inform the rational design of control measures for cholera in Africa.

〔 holera is an acute intestinal infection caused by the bacterium Vibrio cholerae, which produces cholera toxin (CTX), responsible for inducing a rapid and massive loss of body fluids. The seventh cholera pandemic (7P) began in 1961 in Indonesia, before spreading globally, in particular to South Asia (1963), Africa (1970), Latin America (1991), and the Caribbean (i.e., Haiti) (2010) (1). In 2008 to 2012, half a century after the onset of this pandemic, the global burden of cholera was estimated at 1.4 to 4.0 million cases of cholera annually, with 21,000 to 143,000 deaths (2). The agent responsible for the $7 \mathrm{P}$ belongs to the $\mathrm{O} 1$ serogroup (or more rarely, the O139 variant) and the El Tor biotype. Globally, 7P V. cholerae El Tor (7PET) isolates are genetically homogeneous and linked to a single source, the Bay of Bengal in South Asia (3). Phylogenetic analyses have identified at least three indepen- dent, but temporally overlapping, waves of global transmission during the $7 \mathrm{P}(3,4)$.

Africa is the continent most affected by the current pandemic (5); however, little is known about the propagation routes of cholera in this region. Basic epidemiological data from individual countries have provided some insight into cholera dynamics $(6,7)$. Conventional typing methods such as serotyping (Ogawa and Inaba) theoretically provide additional data to discriminate lineages across outbreaks; however, they rapidly proved inadequate or even confusing, and the multiple molecular typing methods used in the pregenomic era (8-13) had low resolution or were generally unsuitable for longer-term phylogenetic inference. Furthermore, even in the genomic era, the use of representative African isolates has been very limited. Previous large-scale genomic studies on the $7 \mathrm{P}(3,4)$ analyzed only 28 African isolates and provided a preliminary view of overall disease transmission on the continent. Other genomic studies dealing with epidemics in Africa have been published, but these studies focused on the disease exclusively at national or regional levels (14-17).

We reconstructed the spatiotemporal spread of cholera across the continent during the $7 \mathrm{P}$ by analyzing the genomes of $1070 \mathrm{~V}$. cholerae $\mathrm{El}$ Tor isolates, including 714 new isolates sequenced in this study (table S1 and fig. S1). The newly sequenced African isolates $(n=569)$ were selected to represent the widest possible temporal and geographic distribution of cases reported to the World Health Organization (WHO) (figs. S2 and S3 and supplementary text note 1). In total, we analyzed the genomes of 6517 PET isolates collected in 45 of 54 African countries between 1966 and 2014 (table S2). Our sampling of cholera isolates in Africa over this time frame is representative of $\sim 1.8$ million (46.8\%) of the $\sim 3.8$ million cases reported from Africa to the WHO between 1970 and 2014 (table S3 and figs. S2 and S3). We note that only a small number of isolates from East and Central Africa from the 1970s and 1980s were available for this study. Consequently, the duration and geographic spread of the imported sublineages (particularly for T3 and T4; see below) may have been underestimated, and some imported sublineages with a limited spatiotemporal spread may have remained undetected. Likewise, low-level and sporadic cases are also likely underrepresented. It is also noteworthy that this study, focusing on 7PET isolates, does not preclude the existence of non-7PET lineage isolates causing sporadic disease in Africa, as has been seen across Latin America [see companion paper by Domman et al. (18)] and in Mozambique (17).

We used two approaches to obtain a robust phylogenetic framework for the inference of propagation routes (19). Maximum likelihood phylogenetic analysis was performed on the 1070 genomes (Fig. 1A and fig. S4), with 9300 single-nucleotide variants (SNVs), evenly distributed over the genome (table S4). We detected a strong temporal signal in the molecular data (fig. S5), allowing us to use a Bayesian phylogenetic approach to provide divergence times for a spatially and temporally representative subset of 228 isolates (Fig. 1B and fig. S6). These robust phylogenies enabled us to infer 11 introductions of 7PET into Africa.

\footnotetext{
${ }^{1}$ Institut Pasteur, Unité des Bactéries Pathogènes Entériques, Paris, 75015, France. ${ }^{2}$ Wellcome Trust Sanger Institute, Wellcome Trust Genome Campus, Hinxton CB10 1SA, UK. ${ }^{3}$ Centers for

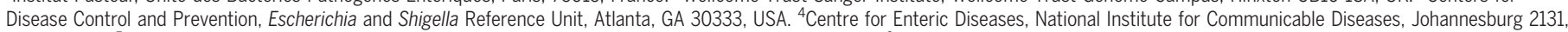
South Africa. ${ }^{5}$ Faculty of Health Sciences, University of the Witwatersrand, Johannesburg 2193, South Africa. ${ }^{6}$ Institut Pasteur, Mathematical Modelling of Infectious Diseases, Paris, 75015 ,

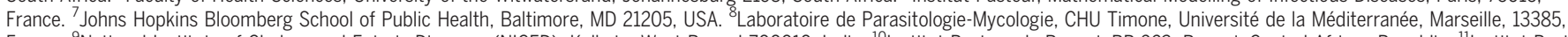

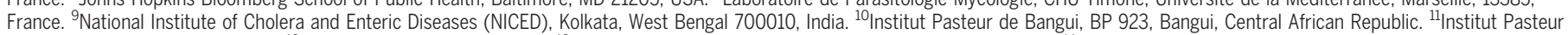
de Dakar, BP 220, Dakar, Senegal. ${ }^{12}$ Epicentre, Paris, 75011, France. ${ }^{13}$ Centre Pasteur du Cameroun, BP 1274, Yaoundé, Cameroon. ${ }^{14}$ Bacteriology and Virology Department, Institut Pasteur,

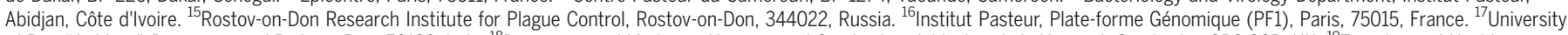
of Bari "A. Moro", Department of Biology, Bari, 70126, Italy. ${ }^{18}$ Department of Medicine, University of Cambridge, Addenbrooke's Hospital, Cambridge CB2 OSP, UK. ${ }^{19}$ Translational Health

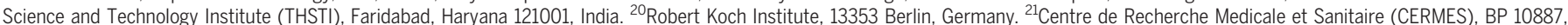
Niamey, Niger. ${ }^{22}$ Médecins Sans Frontières (MSF), Paris, 75011, France. ${ }^{23}$ Cantacuzino National Institute of Research-Development for Microbiology and Immunology, Bucharest, Romania.

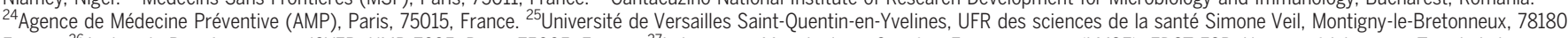
France. ${ }^{26}$ Atelier de Bioinformatique, ISYEB, UMR 7205, Paris, 75005. France. ${ }^{27}$ Laboratoire Microbiologie Santé et Environnement (LMSE), EDST-FSP, Université Libanaise, Tripoli, Lebanon.

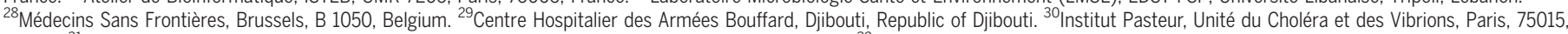
France. ${ }^{31}$ Institut Pasteur, Unité Biodiversité des Bactéries Pathogènes Emergentes, Paris, 75015, France. ${ }^{32}$ Department of Biochemistry and Molecular Biology, Bio21 Molecular Science and Biotechnology Institute, University of Melbourne, Parkville, Victoria 3010, Australia. ${ }^{33}$ London School of Hygiene and Tropical Medicine, London WC1E 7HT, UK.

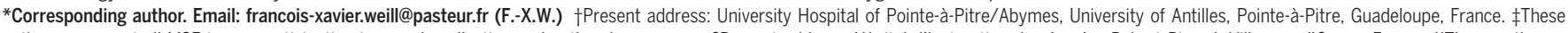

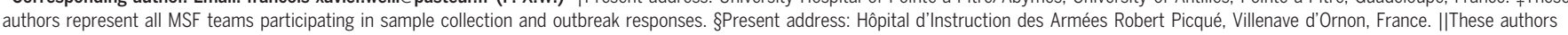
contributed equally to this work.
} 
Each introduction resulted in mid- to long-term spread of cholera (introduction events T1, T3 to T12), and one export from Africa to Peru responsible for the Latin American epidemic in the 1990s [T2 (18)] (Fig. 2, table S5, and supplementary text note 2). All 651 African genomes were assigned to serogroup $\mathrm{O} 1$ on the basis of their $r f b$ region (fig. S7).

We mapped the phylogenetic data onto historical records of African cholera outbreaks. The genomic analysis identified the two "El Tor strains" known to have invaded Africa in 1970: one in West Africa (serotype Ogawa) and the other in East Africa (serotype Inaba).

An "Ogawa strain" was first reported between July and September 1970, in cholera cases in the Black Sea region (Odessa and Kerch) of the former USSR, the Middle East (Lebanon, Israel, Turkey, Jordan), North Africa (Libya and Tunisia), and West Africa (Guinea, Sierra Leone, Liberia, Ghana) (1). This "Ogawa strain" corresponds to our isolates from the $\mathrm{T} 1$ introduction. These initial African $\mathrm{T} 1$ isolates were not directly related to Iraqi isolates from 1966 (the last isolates obtained during the western extension of the $7 \mathrm{P}$ before a lull that ended in 1970) but rather related to three Chinese isolates (1973-1981) and one Indian isolate (1980). This suggests that the resurgence of cholera in 1970 may not have been due to a resumption of westward progression from Iraq, but instead from a new introduction from South or East Asia into Russia and the Middle East. Cholera reached Angola in December 1971 (1). The origin of this outbreak has remained unclear, as (i) it occurred more than $1000 \mathrm{~km}$ from the closest country with a cholera outbreak at this time (Cameroon), and (ii) it was caused by a serotype Inaba strain. Our analysis suggests a West African origin for this outbreak, with $\mathrm{T} 1$ isolates displaying a non-synonymous SNV (G103A) in the wbeT gene. This mutation explains the serotype switch from Ogawa to Inaba (see supplementary text note 2, fig. S8, and tables S1 and S6). This sublineage continued to spread throughout Southern Africa during the 1970s, and it circulated in this region until the early 1990s. These findings are supported by the report of a cholera outbreak in the Mozambican seaport of Beira, imported by air from Angola during late 1973 (20). This particular pattern of long-distance transmission between Angola and Mozambique may be explained by a Portuguese decolonization war involving these two countries at the time. This T1 sublineage was also implicated in a large outbreak in Portugal in 1974, in which 2467 cases and 48 deaths were recorded (21). Colonial troops were stationed uphill from one of the springs supplying the city in which the outbreak began (21). These troops had been traveling back and forth between their base and Angola, Mozambique, and Portuguese Guinea (now Guinea Bissau) (21). All the other Western and Southern European isolates from the early 1970s were found to have originated from West or North Africa.

After the introduction of cholera into West Africa in 1970, we identified multiple subsequent introductions of cholera into this region, with further extensions to the Gulf of Guinea region and the Lake Chad Basin on at least three occasions over the next three decades: T7 (introduction dates: 1982 to 1984), T9 (1988 to 1991), and T12 (2007). The T9 introduction led to outbreaks with high attack rates, such as those that struck Guinea in 1994 (436 reported cholera cases per 100,000 population) and Liberia in 2003 (1241 per 100,000) (table S7).

The second introduction of cholera into Africa in 1970 occurred in East Africa, particularly in Ethiopia in November 1970 (1). The strain involved, another "Inaba strain," corresponds to our T3 isolates with a premature stop codon (C157T)

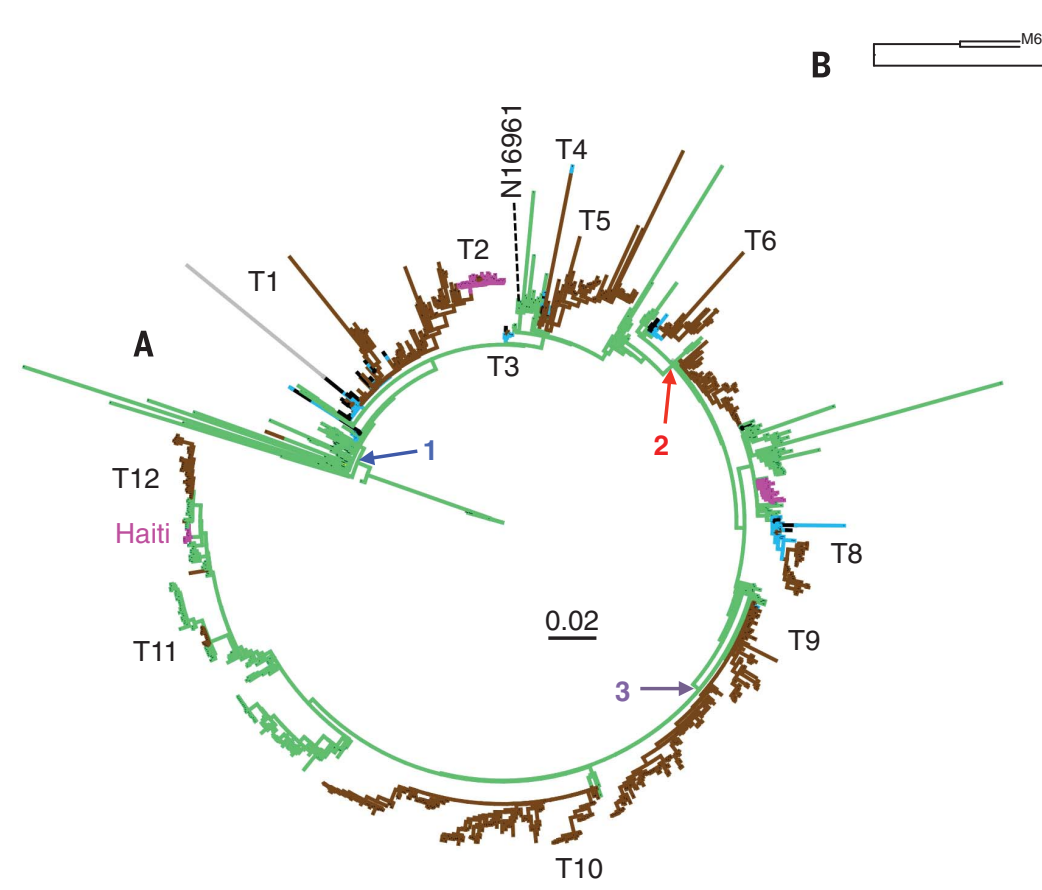

Fig. 1. Phylogeny of seventh pandemic V. cholerae El Tor isolates.

(A) Maximum likelihood phylogeny of the 1070 genomes studied, including M66 as an outgroup. Branches are color-coded according to their geographic location, inferred by stochastic mapping of the geographic origin of each isolate onto the tree. The N16961 reference genome is indicated by a black dashed line. (B) Maximum clade credibility tree produced with BEAST for a subset of 228 representative isolates. The 12 introduction events involving Africa are indicated by the letter T. The three previously described waves (3) are indicated by colored arrows. The clades containing $\mathrm{O} 139$ isolates and that containing isolates from the 2010 outbreak in Haiti are shown.

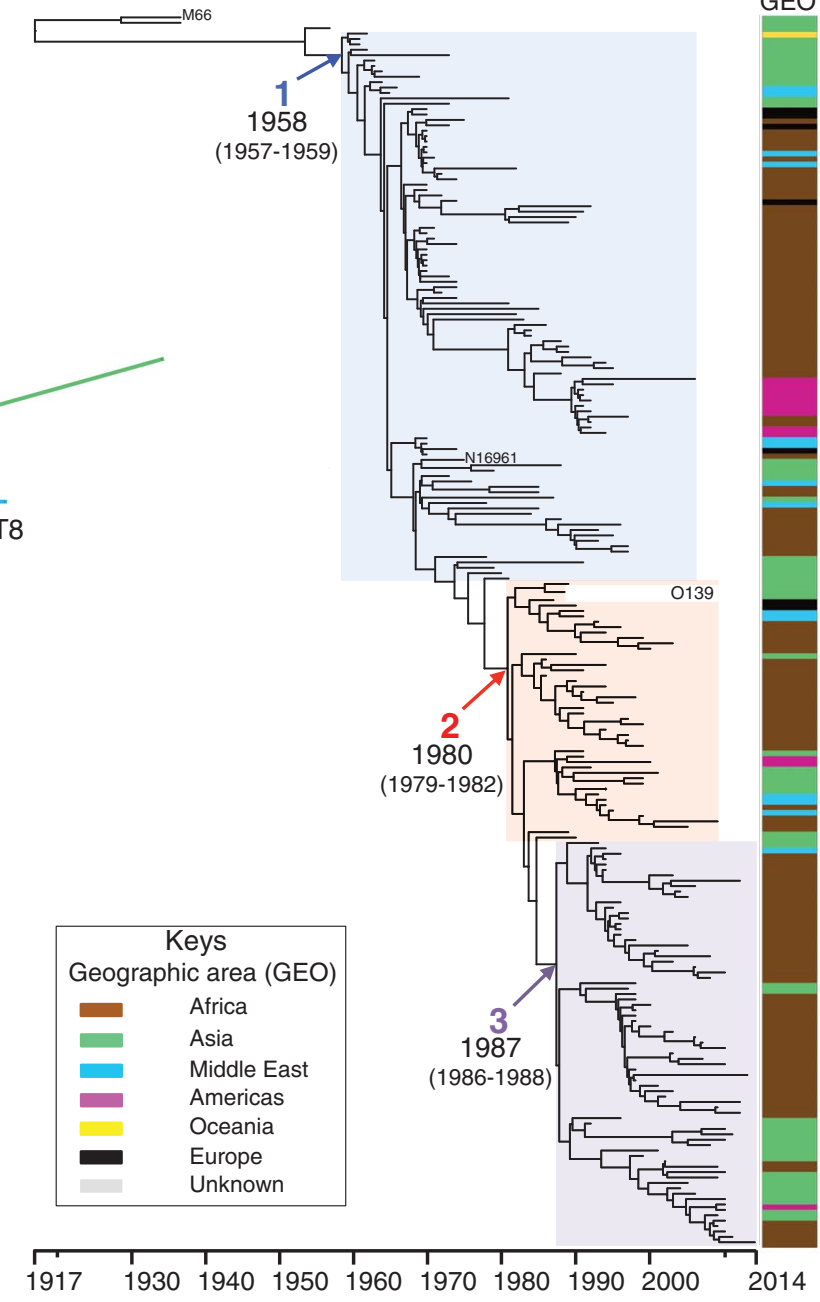



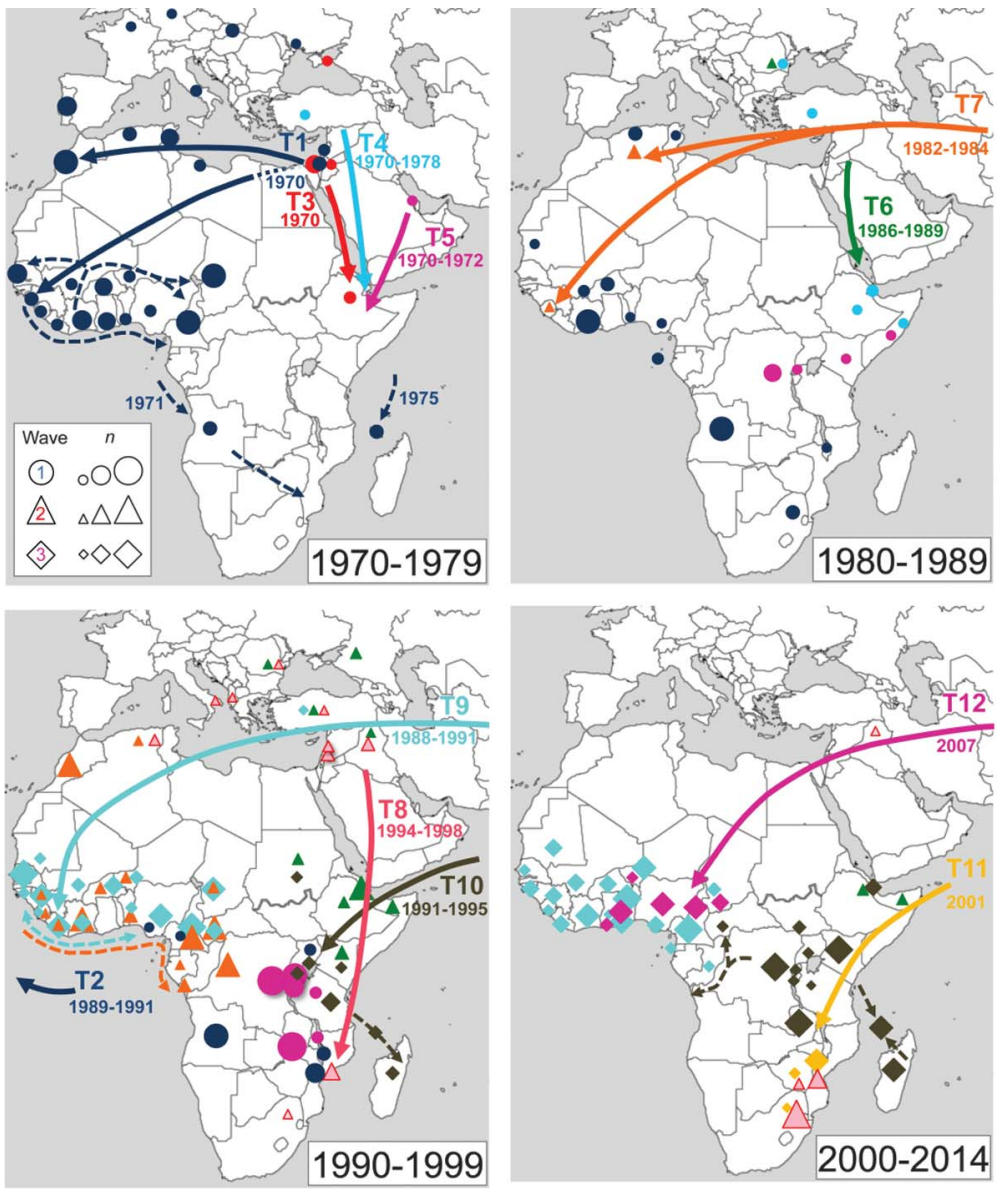

Fig. 2. Inferred propagation routes of seventh pandemic V. cholerae $\mathrm{Ol}$ El Tor populations to, from, and within Africa. The 12 introduction events involving Africa are indicated by the letter T. The date ranges shown for introductions are the median values for the most recent common ancestor (MRCA) in years (taken from BEAST) with the first number indicating the median MRCA of the African isolates and their closest relative from the source location, and the second number indicating the median MRCA of the African isolates. Introductions and inferred secondary transmission chains are indicated by thick and dashed arrows, respectively. Secondary transmission chains for West Africa in 1970 to 1971 are based on published records. The geographic presence of the various lineages is indicated by a circle, triangle, and diamond for waves 1,2 , and 3, respectively, and colored according to the inferred transmission events. The size of the shapes is proportional to the number of genomes analyzed (see fig. S3).

in the wbeT gene (fig. S8 and tables $\mathrm{S} 1$ and $\mathrm{S} 6$ ). The 1970 isolates from Ethiopia are most closely related to isolates from the same year from Jordan and Israel (22) (3- to 9-SNV separation), which is consistent with epidemiological data from the time that suggested an importation from the Middle East (1).

Over the course of the $7 \mathrm{P}$, we identified six other introductions into East Africa (T4, T5, T6, T10) (mostly the Horn of Africa) and Southern Africa (T8, T11) (mostly Mozambique) (Fig. 2). Our data suggest that cholera around the African Great Lakes for a period of $>10$ years was due to the introduction of the T5 and T10 sublineages. The T5 sublineage was, in particular, associated with outbreaks in Rwandan refugee camps in 1994, whereas the T8 sublineage was associated with outbreaks in South Africa in 2001 to 2002 (125,000 cases) and in Zimbabwe in 2008 to 2009
(98,000 cases) (table S7). The T11 sublineage was also found associated with this latter outbreak.

The T1, T3, T4, T5, T6, T8, and, possibly, T9 introduction events involved 7PET sublineages originating from South or East Asia that were circulating in the Middle East before importation into West or East Africa (Figs. 1 and 2). Moreover, epidemiological data for $\mathrm{T} 1$ isolates identified during an outbreak in the Comoros Islands (in the Indian Ocean, off the African coast) in 1975 linked them to pilgrims returning from the Hajj, a religious gathering involving millions of people (20), where cholera outbreaks were frequently described until 1989 (23). The most recent introductions (T10 to T12) appear to be imported directly from South Asia, as no Middle Eastern isolates were found to belong to the imported sublineages. This may be due to (i) an increase in the movement of people between Africa and
South Asia or (ii) a lack of relevant samples, as the most recent Middle Eastern isolate analyzed was collected in 2003.

We observed recurrent patterns of transmission for cholera epidemics in Africa. Separate 7PET sublineages from Asia were repeatedly introduced into two main regions: West Africa and East/Southern Africa. Epidemic waves then propagated regionally, in some instances spreading to Central Africa, over periods of a few years to 28 years (Fig. 3 and table S5). Only two notable instances of sublineage exchange between these two circulation hotspots were identified: (i) the spread of a sublineage between Angola and Mozambique during the Portuguese colonial war in the 1970s (20) and (ii) the spread of a sublineage from the African Great Lakes Region to the western part of the Democratic Republic of the Congo (6) and the Central African Republic via the Congo River and its tributaries in 2011 to 2012 (Fig. 2).

Our study also provides a notable historical perspective on the evolution of antibiotic resistance in 7PET isolates. Antibiotics have been used for decades in the treatment of cholera, as an adjunct to rehydration therapy, as they shorten the duration of diarrhea, thereby limiting bacterial spread $\left[10^{6}\right.$ to $10^{9}$ bacteria per milliliter of stool, with the loss of up to 1 liter of diarrheal fluid per hour (1)].

Our data suggest that African isolates became increasingly resistant to antibiotics over time (figs. S9 to S11). The first antibiotic-resistant African isolates in our collection were recovered in the early 1980 s. No susceptible isolates have been collected since 2000 [96.4\% susceptible isolates between 1970 and 1984 $(n=80 / 83)$, $36.9 \%$ between 1985 and $1999(n=100 / 271)$, and $0 \%$ between 2000 and 2014 $(n=0 / 296)]$. We found that recent patterns of antibiotic resistance among 7PET sublineages in Africa have been largely driven by resistance determinants already present within the genomes of the imported sublineages, rather than by the independent local acquisition of resistance within this region (Fig. 4, A to C, and figs. S9 to S12).

Where it did occur, the local acquisition of antibiotic resistance genes (ARGs) (table S5, fig. S9, supplementary text note 3) mostly involved multidrug-resistant (MDR) plasmids of the IncA/C group. IncA/C plasmids are the only stably maintained plasmids in the 7PET lineage (24). Almost all T5 isolates (1984 to 1998 , wave 1 ) were found to contain an MDR IncA/C plasmid (Fig. 4A and fig. S12). This sublineage, which circulated through the African Great Lakes Region, may be derived from a strain described in Tanzania in 1977. At that time, the Tanzanian Ministry of Health used 1.79 metric tons of tetracycline to control an outbreak caused by a susceptible strain (25). Five months later, $76 \%$ of the isolates were resistant to tetracycline and other antibiotics owing to the acquisition of an IncA/C plasmid (25). We show that IncA/C plasmids were also acquired repeatedly between 1984 and 2014 in T1, T6, and T10 isolates collected in West or East Africa. These acquisition events may also be linked to mass chemoprophylaxis, as observed in Madagascar in 


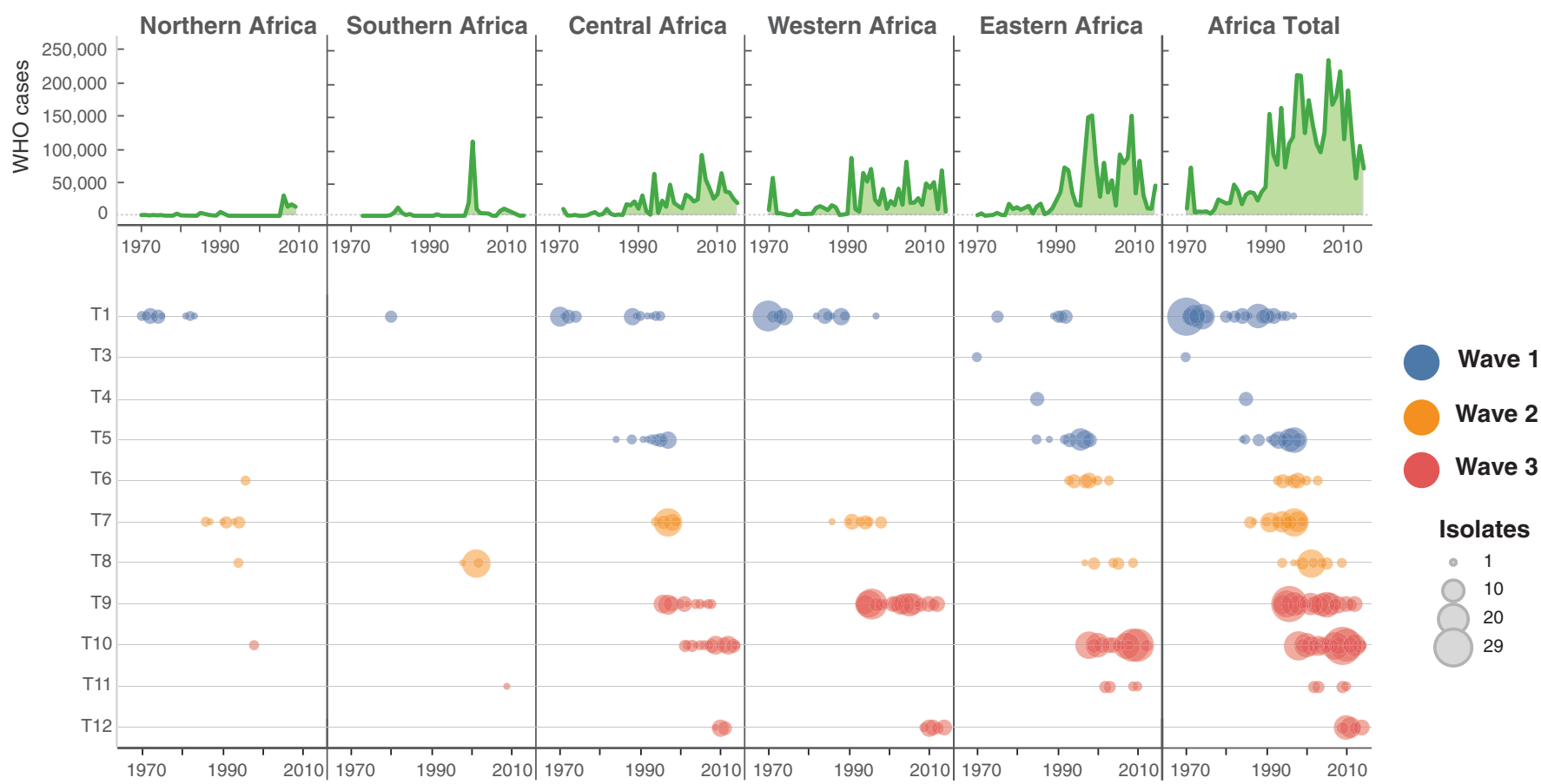

Fig. 3. Geographic and temporal distribution of seventh pandemic V. cholerae El Tor isolates from Africa according to their inferred introduction events (T1 to T12). The annual number of cholera cases reported to the World Health Organization (WHO) is shown in the upper panels. The United Nations subregion scheme was used for the geographic breakdown. The size of the circle scales with the number of genomes analyzed per year.
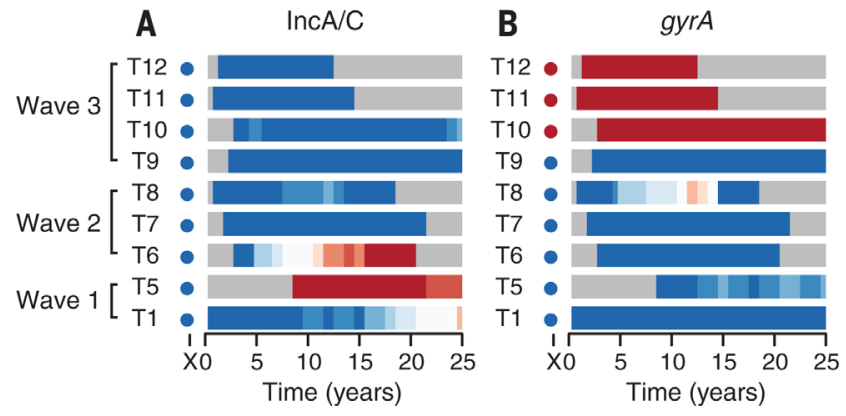

Fig. 4. Evolution of antibiotic resistance in seventh pandemic V. cholerae El Tor isolates from Africa. For each introduction into Africa ( $T 1, T 5$ to $T 12$ ), the proportion of genomes at different time periods following introduction that contain (A) an IncA/C plasmid, (B) a gyrA mutation, and (C) a SXT/R391 genomic island. (D) For wave 3, the mean number of antibiotic resistance genes (ARGs) per isolate at different time periods following introduction. Each value is calculated over a 10-year window,
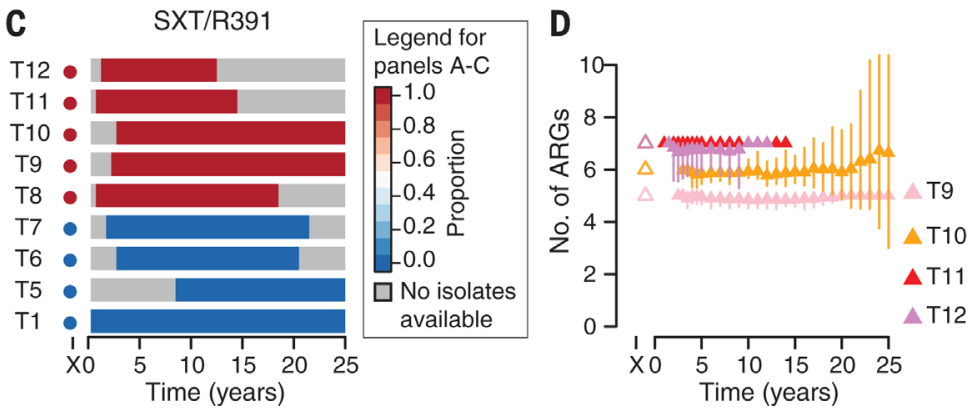

with the point plotted at the midpoint of the period. Sampling bias was prevented by randomly down-sampling the number of sequences to one sequence per country per year. $\ln (A),(B)$, and $(C)$, the color in the leftmost circle (at the value " $X$ " on the $x$ axis) represents the value for the closest genomic sequence obtained from outside Africa [for (A) to (C), a value of 1 implies that the sequence included the corresponding antibiotic resistance determinant, whereas 0 implies that the sequence lacked the determinant].
1999 (T10), when doxycycline was used for prophylaxis (26). The acquisition of resistance to the quinolone nalidixic acid, mediated by various point mutations in the DNA gyrase gene, gyrA, was identified three times in Central and East Africa (Fig. 4B, figs. S10 to S12, and table S1). The appearance of two independent mutations [resulting in the substitution of $\mathrm{Ser}^{83}$ with $\mathrm{Arg}$ (S83R) and $\mathrm{Asp}^{87}$ with $\mathrm{Tyr}(\mathrm{D} 87 \mathrm{Y})$ ] in T5 isolates may reflect the heavy use of nalidixic acid for the treatment of dysentery in Rwandan refugee camps, which experienced concomitant outbreaks of diarrhea caused by Shigella dysenteriae type 1 and $V$. cholerae $\mathrm{O} 1$ at this time (27).
Since 2000, almost all antibiotic-resistant 7PET sublineages circulating in Africa (T8 to T12) originated from South Asia and carry their ARGs on genomic islands SXT/R391 (28) or GI-15 (29) (Fig. 4C, figs. S6 and S11, table S5, and supplementary text note 3 ). These more recently imported sublineages also carry resistance mutations in gyr $A$ [resulting in the replacement of $\mathrm{Ser}^{83}$ with Ile (S83I); all T10 to T12 isolates] and an additional mutation in parC [resulting in the substitution of $\mathrm{Ser}^{85}$ with Leu (S85L)] that decrease susceptibility to ciprofloxacin (all T11 and T12 isolates). The T9 to T12 isolates, which contain a similar SXT/R391 element (ICEVchInd5/ICEVchBan5), do not con- tain tetracycline resistance genes. The ARG content of these SXT/R391-containing sublineages has remained very stable for more than a decade since their introduction into Africa (Fig. 4D, fig. S12, and table S1). The apparent incompatibility between SXT/R391 and IncA/C plasmids in 7PET may be due to functional interference between these related elements (28).

Our data demonstrate that the repeated introductions of 7PET sublineages into Africa describe the burden of disease (as measured by cases reported to the WHO) seen across this continent. These data are consistent with epidemiological studies, which have demonstrated that human-related 
factors play a much more important role in cholera dynamics in Africa than climatic and environmental factors $(6,7)$. Our data do not suggest that aquatic environmental reservoirs are the primary source of epidemic cholera in Africa, as has been suggested (30). Instead, these results highlight the role that humans play in the long-term spread and maintenance of the pathogen, whether by direct (human-to-human) or indirect (pollution of the environment with feces from cholera patients) transmission. Undoubtedly, the factors influencing the epidemiology and transmission of cholera are complex, but these data provide a detailed genetic context against which we can gauge the impact of interventions on future patterns of disease in this region.

\section{REFERENCES AND NOTES}

1. D. Barua, W. B. Greenough, Eds., Cholera (Plenum, New York, 1992)

2. M. Ali, A. R. Nelson, A. L. Lopez, D. A. Sack, PLOS Negl. Trop. Dis. 9, e0003832 (2015)

3. A. Mutreja et al., Nature 477, 462-465 (2011).

4. X. Didelot et al., PLOS Genet. 11, e1005072 (2015).

5. N. H. Gaffga, R. V. Tauxe, E. D. Mintz, Am. J. Trop. Med. Hyg. 77, 705-713 (2007)

6. S. Rebaudet, B. Sudre, B. Faucher, R. Piarroux, J. Infect. Dis. 208 (suppl. 1), S46-S54 (2013)

7. S. Rebaudet, B. Sudre, B. Faucher, R. Piarroux, J. Infect. Dis. 208 (suppl. 1), S98-S106 (2013).

8. S. Koblavi, F. Grimont, P. A. D. Grimont, Res. Microbiol. 141 645-657 (1990).

9. D. K. Karaolis, R. Lan, P. R. Reeves, J. Bacteriol. 176, 6199-6206 (1994).
10. G. M. Evins et al., J. Infect. Dis. 172, 173-179 (1995).

11. R. Lan, P. R. Reeves, J. Clin. Microbiol. 40, 172-181 (2002).

12. C. Lam, S. Octavia, P. Reeves, L. Wang, R. Lan, Emerg. Infect. Dis. 16, 1130-1132 (2010)

13. C. Lam, S. Octavia, P. R. Reeves, R. Lan, BMC Microbiol. 12, 82 (2012).

14. J. Kiiru et al., PLOS ONE 8, e74829 (2013).

15. S. Rebaudet et al., PLOS Negl. Trop. Dis. 8, e2898 (2014).

16. R. S. Kaas et al., PLOS ONE 11, e0155691 (2016)

17. M. Garrine et al., PLOS Negl. Trop. Dis. 11, e0005671 (2017).

18. D. Domman et al., Science 358, XXX (2017).

19. Materials and methods are available as supplementary materials.

20. M. Echenberg, Ed., Africa in the Time of Cholera: A History of Pandemics from 1817 to the Present (Cambridge Univ. Press, Cambridge, UK, 2011)

21. P. A. Blake et al., Am. J. Epidemiol. 105, 337-343 (1977).

22. J. Cohen et al., Lancet 298, 86-89 (1971).

23. Q. A. Ahmed, Y. M. Arabi, Z. A. Memish, Lancet 367 , 1008-1015 (2006).

24. K. Rahal, G. Gerbaud, D. H. Bouanchaud, Ann. Microbiol. (Paris) 129, 409-414 (1978).

25. K. J. Towner, N. J. Pearson, F. S. Mhalu, F. O'Grady, Bull. World Health Organ. 58, 747-751 (1980).

26. J. A. Dromigny et al., Emerg. Infect. Dis. 8, 336-338 (2002).

27. E. Njamkepo et al., Nat. Microbiol. 1, 16027 (2016)

28. R. A. Wozniak et al., PLOS Genet. 5, e1000786 (2009).

29. C. J. Grim et al., J. Bacteriol. 192, 3524-3533 (2010).

30. R. R. Colwell, Science 274, 2025-2031 (1996).

\section{ACKNOWLEDGMENTS}

This study was supported by the Institut Pasteur and the Institut Pasteur International Network, the Institut de Veille Sanitaire, the French government's Investissement d'Avenir program,

Laboratoire d'Excellence "Integrative Biology of Emerging

Infectious Diseases" (grant no. ANR-10-LABX-62-IBEID), the

Fondation Le Roch-Les Mousquetaires, the Wellcome Trust through grant 098051 to the Sanger Institute, and the Indian Council of Medical Research, New Delhi, India. The findings and conclusions in this report are those of the authors and do not necessarily represent the official position of the Centers for Disease Contro and Prevention. We thank C. Bréchot and H.-F. Thiéfaine for their support; O. Vandenberg, Y. Germani, and M.-C. Fonkoua for providing isolates; D. Nedelec and E. E. Hansen for helpful discussions; S. Dupke and A. Smith for technical assistance; L. Ma, M. Santovenia, J. Winkjer, M. Aslett, A. J. Page, J. Keane, and the sequencing teams at the Institut Pasteur, the Wellcome Trust Sanger Institute, and the Centers for Disease Control and Prevention for sequencing the samples. F.J.L. and M.-L.Q. are members of the WHO Global Task Force on Cholera Control. F.J.L. is a past member of the WHO Strategic Advisory Group of Experts working group for cholera vaccines. J.P. is a member of the Institut Pasteur Scientific Council. J.P. has received consulting fees from Specific Technologies, Mountain View, California. Short-read sequences have been deposited in the European Nucleotide Archive (ENA) (www.ebi.ac.uk/ena) under study accession numbers PRJEB8764, PRJEB2215, and PRJEB19893. The whole-genome alignment for the 1070 genomes and other files have been deposited in FigShare: https://figshare. $\mathrm{com} / \mathrm{s} / \mathrm{d} 6 \mathrm{clc6f02eac0c9c871e.} \mathrm{Phylogeny} \mathrm{and} \mathrm{metadata} \mathrm{can} \mathrm{be}$ viewed interactively at https://microreact.org/project/ globalcholera?tt=rc

\section{SUPPLEMENTARY MATERIALS}

www.sciencemag.org/content/358/6364/785/suppl/DC1

Materials and Methods

Supplementary Text

Figs. S1 to S12

Tables S1 to S9

References (31-77)

26 June 2017; accepted 10 October 2017

10.1126/science.aad5901 


\section{Science}

\section{Genomic history of the seventh pandemic of cholera in Africa}

François-Xavier Weill, Daryl Domman, Elisabeth Njamkepo, Cheryl Tarr, Jean Rauzier, Nizar Fawal, Karen H. Keddy, Henrik Salje, Sandra Moore, Asish K. Mukhopadhyay, Raymond Bercion, Francisco J. Luquero, Antoinette Ngandjio, Mireille Dosso, Elena Monakhova, Benoit Garin, Christiane Bouchier, Carlo Pazzani, Ankur Mutreja, Roland Grunow, Fati Sidikou, Laurence Bonte, Sébastien Breurec, Maria Damian, Berthe-Marie Njanpop-Lafourcade, Guillaume Sapriel, Anne-Laure Page, Monzer Hamze, Myriam Henkens, Goutam Chowdhury, Martin Mengel, Jean-Louis Koeck, Jean-Michel Fournier, Gordon Dougan, Patrick A. D. Grimont, Julian Parkhill, Kathryn E. Holt, Renaud Piarroux, Thandavarayan Ramamurthy, Marie-Laure Quilici and Nicholas R. Thomson

Science 358 (6364), 785-789.

DOI: $10.1126 /$ science.aad5901

\section{Wave upon wave of disease}

The cholera pathogen, Vibrio cholerae, is considered to be ubiquitous in water systems, making the design of eradication measures apparently fruitless. Nevertheless, local and global Vibrio populations remain distinct. Now, Weill et al. and Domman et al. show that a surprising diversity between continents has been established. Latin America and Africa bear different variants of cholera toxin with different transmission dynamics and ecological niches. The data are not consistent with the establishment of long-term reservoirs of pandemic cholera or with a relationship to climate events. Science, this issue p. 785 , p. 789

ARTICLE TOOLS

SUPPLEMENTARY

MATERIALS

RELATED
CONTENT

REFERENCES

PERMISSIONS http://science.sciencemag.org/content/358/6364/785

http://science.sciencemag.org/content/suppl/2017/11/09/358.6364.785.DC1

http://science.sciencemag.org/content/sci/358/6364/706.full http://science.sciencemag.org/content/sci/358/6364/789.full http://stm.sciencemag.org/content/scitransmed/5/192/192ra86.full

This article cites 74 articles, 11 of which you can access for free http://science.sciencemag.org/content/358/6364/785\#BIBL

http://www.sciencemag.org/help/reprints-and-permissions

Use of this article is subject to the Terms of Service

Science (print ISSN 0036-8075; online ISSN 1095-9203) is published by the American Association for the Advancement of Science, 1200 New York Avenue NW, Washington, DC 20005. 2017 (C) The Authors, some rights reserved; exclusive licensee American Association for the Advancement of Science. No claim to original U.S. Government Works. The title Science is a registered trademark of AAAS. 